\title{
Larvas de Philornis deceptiva Dodge \& Aitken (Diptera, Muscidae) no Sanhaçu-cinzento Thraupis sayaca (Linnaeus 1766) (Thraupidae) no município de Seropédica, Rio de Janeiro, Brasil
}

\author{
Hermes Ribeiro Luz $^{1,3}$, Ildemar Ferreira ${ }^{1}$ \& Márcia Souto Couri ${ }^{2}$ \\ ${ }^{1}$ Laboratório de Ornitologia, Departamento de Biologia Animal, Instituto de Biologia, \\ Universidade Federal Rural do Rio de Janeiro - UFRRJ, \\ Km 47, CEP 23890-000, Seropédica, RJ, Brasil, e-mail: ferreira@ufrrj \\ ${ }^{2}$ Departamento de Entomologia, Museu Nacional, Universidade Federal Rural do Rio de Janeiro - UFRJ, \\ Quinta da Boa Vista, s/n, CEP 20940-040, São Cristóvão, RJ, Brasil \\ ${ }^{3}$ Autor para correspondência: Hermes Ribeiro Luz,e-mail: hermes@ufrrj.br
}

LUZ, H.R., FERREIRA, I. \& COURI, M.S. 2008. Larvae of Philornis deceptiva Dodge \& Aitken (Diptera, Muscidae) on the Sayaca Tanager Thraupis sayaca (Linnaeus 1766) (Thraupidae) in the municipality of Seropédica, Rio de Janeiro, Brazil. Biota Neotrop. 8(2): http://www.biotaneotropica.org.br/v8n2/en/ abstract?short-communication+bn01908022008.

\begin{abstract}
Six nestlings of the Sayaca Tanager, Thraupis sayaca sustained 42 larvae of Philornis deceptiva during its breeding period from August to October, 2006 in the municipality of Seropédica, Rio de Janeiro. Larvae were observed since the $3^{\text {rd }}$ day after hatching. They were taken off and put in an artificial nest into plastic box and their life cycle observed until emergence. Thirty-two larvae reached adult stage and were preserved in alcohol $70 \%$ for further identification. Four nestlings died before reaching adult stage. Four nestlings died before reaching adult stage. They were hard infesting by Philornis larvae. In this study we presented Thraupis sayaca as a new host for Philornis deceptiva.

Keywords: myiasis, parasitology, Neotropical region.
\end{abstract}

LUZ, H.R., FERREIRA, I. \& COURI, M.S. 2008. Larvas de Philornis deceptiva Dodge \& Aitken (Diptera, Muscidae) no Sanhaçu-cinzento Thraupis sayaca (Linnaeus 1766) (Thraupidae) no município de Seropédica, Rio de Janeiro, Brasil. Biota Neotrop. 8(2): http://www.biotaneotropica.org.br/v8n2/pt/abstract?shortcommunication+bn01908022008.

Resumo: Seis ninhegos do Sanhaçu-cinzento, Thraupis sayaca albergaram 42 larvas de Philornis deceptiva durante o período reprodutivo, de agosto a outubro de 2006, no município de Seropédica, Rio de Janeiro. As larvas foram observadas a partir do $3^{\circ}$ dia após a eclosão e colocadas em um recipiente plástico sendo seu ciclo de vida acompanhado até que emergissem. Trinta e duas larvas atingiram a fase adulta e foram preservadas em álcool a 70\%, para posterior identificação. Quatro ninhegos morreram antes de atingir a idade adulta. Eles estavam intensamente infestados por larvas de Philornis. No presente estudo apresentamos Thraupis sayaca como novo hospedeiro para Philornis deceptiva.

Palavras-chave: miíases, parasitologia, região neotropical. 


\section{Introdução}

A associação de Philornis com diversas aves do neotrópico é um fato bem conhecido, tendo sido registrado, provavelmente, no século XVII por Marcgrave em 1648, naturalista integrante da Corte de Maurício de Nassau-Siegen, durante o domínio holandês, no nordeste do Brasil (Teixeira 1987). A associação de Philornis com os hospedeiros pode ser classificada como coprófaga, semi-hematófaga e hematófaga intra-dérmica (Couri 1985). Philornis distribui-se por toda a região neotropical sendo que somente duas espécies ocorrem no sul dos Estados Unidos, Philornis porteri Dodge, 1955 e Philornis angustifrons Loew, 1861 (Couri 1984). Pont (1972) catalogou 33 espécies na região Neotropical, às quais foram adicionadas 14 por Couri (1983a, b). Segundo Couri (1984) a literatura cita 23 espécies do gênero Philornis relacionadas com filhotes de 75 espécies de aves, sendo dois casos em aves adultas registrados por Macquart (1854) e Dodge \& Atiken (1968). No entanto, Teixeira (1999) mencionou o parasitismo por Philornis para 105 espécies pertencentes a 32 famílias diferentes de aves. Dentre estas descreve sanhaçu-cinzento Thraupis sayaca parasitada por Philornis sp. sem mencionar a espécie do parasita.

No presente trabalho, apresentamos o primeiro registro de larvas de Philornis deceptiva (Díptera, Muscidae) em ninhegos de Thraupis sayaca (Aves, Passeriformes, Thraupidae) no município de Seropédica, Rio de Janeiro.

O Sanhaçu-cinzento, Thraupis sayaca é um dos pássaros mais abundantes do Brasil oriental. Vive nas árvores em qualquer lugar, tanto em campos ou áreas de cultivo do interior como em cidades (Sick 1997). Alimenta-se de frutos, folhas, brotos, flores de eucalipto (Eucalyptus spp.) e insetos (Höfling \& Camargo 1996).

\section{Materiais e Métodos}

Entre os meses de agosto a outubro de 2006, a equipe de Ornitologia da Universidade Federal Rural do Rio de Janeiro, acompanhou a biologia reprodutiva do sanhaço-cinzento, Thraupis sayaca na Baixada Fluminense, estado do Rio de Janeiro. Os ninhegos foram retirados do ninho para a tomada das medidas morfométricas e tratamento de miíases sendo posteriormente marcados (com anilhas coloridas) e recolocados no ninho.

Foram estudados, no total, seis ninhegos de T. sayaca. Estes foram medidos e pesados sempre antes da retirada das larvas, para que pudéssemos ter um dado preciso do exemplar parasitado.

As larvas foram coletadas com auxílio de pinças em diferentes regiões do corpo. Logo após a retirada foram colocadas em um recipiente de plástico desinfetado e recoberto com tecido de filó, o mesmo ocorrendo com pupas encontradas no interior do ninho logo depois do abandono do mesmo pelos ninhegos. De cada ninhego, foram removidas até 8 larvas para posterior identificação do parasita. No fundo de cada pote colocou-se capim seco e uma fina camada de terra, para permitir a pupação e o término da metamorfose. Após a emergência de formas adultas dos dípteros, colocamos no interior de cada pote um algodão umedecido com água, permitindo assim a manutenção da umidade do ambiente.

As formas adultas não foram sacrificadas sendo coletadas após sua morte natural, para que tivéssemos uma estimativa do tempo de vida dos parasitos em cativeiro. À medida que os adultos iam morrendo eram coletados e conservados em etanol $70^{\circ} \mathrm{GL}$ para posterior identificação. As larvas que não empuparam e as pupas que não geraram adultos foram depositadas no Laboratório de Entomologia do Museu Nacional do Rio de Janeiro.

\section{Resultados e Discussão}

Não há registro da espécie de Philornis deceptiva parasitando ninhegos de Thraupis sayaca (Linnaeus 1766). Teixeira (1999) men- ciona o parasitismo em Thraupis sayaca por larvas de Philornis, mas sem citar a espécie do parasita. Foram retiradas um total de 42 larvas, onde $32(76 \%)$ se tornaram adultos sendo posteriormente identificados como Philornis deceptiva Dodge \& Aitken.

As regiões mais infestadas por larvas de $P$. deceptiva foram: asa, cabeça, pescoço e alto dorso. Não podemos constatar em nosso trabalho o que, provavelmente, teria atraído estes muscideos, já que não observamos em nenhum dos ninhos estudados $(\mathrm{n}=2)$ acúmulo de fezes no interior dos ninhos para que possa atraí-los. Segundo Teixeira (1990) fêmeas de P. rufoscutellaris aptas para a postura podem buscar sítios propícios para ovoposição, sendo estas atraídas pelo forte odor de amônia despendido pela matéria orgânica depositada no interior dos ninhos. Acreditamos que as espécies do gênero Philornis não possuem especificidade por hospedeiros, podendo assim, haver outros fatores atuando nesta atração a ninhegos do presente estudo.

Dos seis ninhegos estudados quatro chegaram ao óbito, estes estavam mais infestados e um pouco mais leves que os sobreviventes. Provavelmente o baixo peso oriundo do parasitismo e a alta infestação, em relação aos dois sobreviventes, tiveram uma ação direta na morte dos ninhegos $(\mathrm{n}=4)$. Higgins et al. (2005) descrevem a morte de ninhegos de $S$. islelorum devido ao acentuado parasitismo por larvas de Philornis. Couri (1985) descreveu a presença de 32 larvas de Philornis sp. no bem-te-vi, chegando a deformá-lo, porém sem ocasionar sua morte. Aguilar et al. (1999) registram ninhegos de L. euleri parasitados por larvas de Philornis onde mesmo após a retirada de todas as larvas os ninhegos vieram a óbito, sendo que no ano seguinte em um novo estudo com a mesma espécie optaram por não retirar nenhuma larva dos hospedeiros, chegando todos a óbito dias depois.

\section{Referências Bibliográficas}

AGUILAR, T.M., LEITE, L.O. \& MARINI, M.A. 1999. Biologia da nidificação de Lathrotriccus euleri (Cabanis 1968) (Tyrannidae) em fragmentos de mata de Minas Gerais. Revista Brasileira de Ornitologia 7(2):125-133.

ARENDT, W.J. 1985. Philornis ectoparasitism of Pearly-eyed Trashers. II. Effects on adults and reproduction. The Auk 102(2):281-292.

COURI, M.S. 1984. Notes and description of Philornis flies (Diptera: Muscidae). Cyrtoneurinae. Revista Brasileira de Entomologia 28(4):473-490.

COURI, M.S. 1985. Considerações sobre as relações ecológicas das larvas de Philornis Meinert, 1890 (Diptera: Muscidae) com aves. Revista Brasileira de Entomologia 29(1):17-20.

DODGE, H.R. \& AITKEN, T.H.G. 1968. Philornis flies from Trinidad (Díptera: Muscidae) Ibid. 41:134-154.

HIGGINS, B.F., LOPES, L.E., SANTANA, F.H.A., COURI, M. \& PUJOL-LUZ, J.R. 2005. Sobre a ocorrência de Philornis angustifrons e P. deceptiva (diptera: muscidae) em ninhos de Suiriri affinis e S. islerorum (Aves, Tyrannidae), no cerrado do Distrito Federal, Brasil. Revista Entomologia y Vectores, 12(1):127-131.

HOFLING, E. \& CAMARGO, H.F.A. 1996. Aves no Campus. 2 ed. Editora da Universidade de São Paulo, SP. p. 84.

MACQUART, J. 1854. Notice sur une nouvelle espèce d Aricie, diptère de la tribu des Anthomyzides. Ann. Soc. Entomol. Fr. 3(1):657-660.

PONT, A.C. 1972. Family Muscidae. Volume 97:1-111. In A catalogue of the Diptera of the Americas South of the United States. Museu de Zoologia, Universidade de São Paulo.

POSSATI, M., LEMOS, M., PINTO, C., MELLO, R.P., GUERIM, L \& SERRA-FREIRE, N.M. 2001. Ocorrência de Philornis bella Couri, 1984 em Pitangus sulphuratus (Sclater 1888) (Aves Tyrannidae) em Niterói estado do Rio de Janeiro, Brasil. Revista Entomologia y Vectores 8(3):391-394.

SICK, H. 1997. Ornitologia Brasileira. Editora Nova Fronteira, Rio de Janeiro. 
SMITH, N. 1968. The advantage of being parasitized. Nature 219(5155): 690-694.

TEIXEIRA, D.M. 1987. As fontes do paraíso. Um ensaio sobre a ornitologia no Brasil holandês (1624-1654). Dissertação de Mestrado, Universidade Federal do Rio de Janeiro, RJ.

TEIXEIRA, D.M. 1990. Notas sobre a biologia de Philornis rufoscutellaris Couri, 1983 (Diptera: Muscidae) e sua associação com ninhos de aves. Revista Brasileira de Entomologia 34(2):271-275.
TEIXEIRA, D.M. 1999. Myiasis caused by obligatory parasites. General observations on the biology of species of the genus Philornis Meinert, 1890 (Diptera Muscidae) p. 71-96. In Myiasis in Man and Animals in the Neotropical Region - Bibliographic Database (J. H. Guimarães e N. Papavero, ed.). FAPESP, Editora Plêiade, São Paulo. 308 p.

Recebido em 18/10/07 Versão Reformulada recebida em 08/05/08 Publicado em 20/05/08 